\title{
CT Evaluation for Clinical Lung Cancer Staging: Do Multiplanar Measurements Better Reflect Pathologic T-Stage than Axial Measurements?
}

\author{
Sohee Park, $M D^{1}$, Sang Min Lee, $M D^{1}$, Jooae Choe, $M D^{1}$, June-Goo Lee, $P h D^{2}$, Sang Min Lee, MD', \\ Kyung-Hyun Do, $\mathrm{MD}^{1}$, Joon Beom Seo, $\mathrm{MD}^{1}$ \\ ${ }^{1}$ Department of Radiology and Research Institute of Radiology, University of Ulsan College of Medicine, Asan Medical Center, Seoul, Korea; \\ ${ }^{2}$ Department of Convergence Medicine, University of Ulsan College of Medicine, Asan Medical Center, Seoul, Korea
}

Objective: To retrospectively investigate whether tumor size assessment on multiplanar reconstruction (MPR) CT images better reflects pathologic T-stage than evaluation on axial images and evaluate the additional value of measurement in threedimensional (3D) space.

Materials and Methods: From 1661 patients who had undergone surgical resection for primary lung cancer between June 2013 and November 2016, 210 patients (145 men; mean age, 64.4 years) were randomly selected and 30 were assigned to each pathologic T-stage. Two readers independently measured the maximal lesion diameters on MPR CT. The longest diameters on 3D were obtained using volume segmentation. T-stages determined on CT images were compared with pathologic T-stages (overall and subgroup-Group 1, T1a/b; Group 2, T1c or higher), with differences in accuracy evaluated using McNemar's test. Agreement between readers was evaluated with intraclass correlation coefficients (ICC).

Results: The diagnostic accuracy of MPR measurements for determining T-stage was significantly higher than that of axial measurement alone for both reader $1(74.3 \%$ [156/210] vs. 63.8\% [134/210]; $p=0.001)$ and reader $2(68.1 \%[143 / 210]$ vs. $61.9 \%[130 / 210] ; p=0.049)$. In the subgroup analysis, diagnostic accuracy with MPR diameter was significantly higher than that with axial diameter in only Group $2(p<0.05)$. Inter-reader agreements for the ICCs on axial and MPR measurements were 0.98 and 0.98 . The longest diameter on 3D images showed a significantly lower performance than MPR, with an accuracy of $54.8 \%(115 / 210)(p<0.05)$.

Conclusion: Size measurement on MPR CT better reflected the pathological T-stage, specifically for T1c or higher stage lung cancer. Measurements in a 3D plane showed no added value.

Keywords: Lung; Neoplasm staging; Multidetector computed tomography

\section{INTRODUCTION}

In the new eighth edition of the lung cancer staging system, the T descriptor is further subdivided, and every centimeter difference can result in a change in the tumor stage (1). Therefore, precise and consistent measurement of lesion size on CT has become critical for preoperative clinical staging of lung cancer.

Since the pathologic T-stage is theoretically determined by the longest diameter of the lesion on the resected specimen, size measurement on $\mathrm{CT}$ needs to be performed using multiplanar reconstructions (MPR) to accurately

Received November 25, 2018; accepted after revision March 27, 2019.

This study was supported funding by a grant (2018-7014) from the Asan Institute for Life Sciences, Asan Medical Center, Seoul, Korea and by Basic Science Research Program through the National Research Foundation of Korea (NRF) funded by the Ministry of Science, ICT \& Future Planning (grant number: NRF-2016R1A2B1016355).

Corresponding author: Sang Min Lee, MD, Department of Radiology and Research Institute of Radiology, University of Ulsan College of Medicine, Asan Medical Center, 88 Olympic-ro 43-gil, Songpa-gu, Seoul 05505, Korea.

- Tel: (822) 3010-4382 • Fax: (822) 3010-6645• E-mail: sangmin.lee.md@gmail.com

This is an Open Access article distributed under the terms of the Creative Commons Attribution Non-Commercial License (https:// creativecommons.org/licenses/by-nc/4.0) which permits unrestricted non-commercial use, distribution, and reproduction in any medium, provided the original work is properly cited. 
reflect tumor size. In this regard, the Fleischner Society has emphasized that MPR in the coronal and sagittal planes should be used when assessing nodule size, in addition to transverse plane images (2). Interestingly, the Fleischner Society does not recommend oblique reformations because of concerns regarding the ability to reproduce the same degree of obliquity during follow-up. However, the diameter in three-dimensional (3D) space containing the longest axis of the 3D tumor volume may show the best correlation with the measurement of the longest dimension of the resected specimen in pathologic examinations.

Several studies have been conducted to determine an accurate and coherent method for tumor size measurement (3-6). Heidinger et al. (4) reported that for solid lung adenocarcinomas $\leq 30 \mathrm{~mm}$, the long-axis CT diameter better reflects the pathologic T-stage than the average of the long and short diameters. Ridge et al. (6) showed that combining MPR measurement with axial measurement of lung cancers resulted in upstaging of T-stage by about $20 \%$ for two readers using the seventh edition of the lung cancer staging system. However, the application of MPR measurement led to slightly decreased agreement between CT-defined and pathologic stages, and between observers.

Recently, using the eighth edition of the lung cancer staging system, Ahn et al. (7) demonstrated that MPR measurement was similar to axial measurements in terms of diagnostic accuracy and interobserver agreement. However, they included only subsolid nodules of pathologically confirmed T1 stages.

Since MPR measurements in routine lung cancer workup require more time and effort, the potential diagnostic improvements should be verified in a large population including all the various T-stages in the eighth edition of the lung cancer staging system; this would facilitate wide acceptance of MPR measurements. Therefore, the purpose of this study was to retrospectively investigate whether tumor size assessment on MPR CT images better reflects the pathologic T-stage than evaluation on axial images and to evaluate the additional value of measurements in 3D space.

\section{MATERIALS AND METHODS}

This retrospective study was approved by the Institutional Review Board of our center, which waived the requirement for patients' informed consent (approval number: 20180519).

\section{Study Population}

A retrospective search of the electronic medical records of our hospital between June 2013 and November 2016 was performed by one radiologist (with 2 years of experience in chest (T). A total of 1661 patients who had undergone preoperative chest $\mathrm{CT}$ within 30 days before surgical resection of primary lung cancer were identified.

Patients were enrolled on the basis of the following criteria: 1) available thin-section axial chest CT data (slice thickness, 1-1.25 mm) that could be reformatted to sagittal and coronal planes $(2,8-11) ; 2)$ lung cancers manifesting as solid nodules on $\mathrm{CT}$; and 3 ) no history of neoadjuvant chemotherapy or radiotherapy. Lung cancers that were not clearly marginated due to accompanying lung parenchymal collapse or a central location were excluded by two radiologists (with 2 and 16 years' experience in chest $\mathrm{CT}$, respectively) in consensus.

From the 1250 patients identified as meeting the study criteria, 30 were randomly selected from each pathologic T-stage (T1a, T1b, T1c, T2a, T2b, T3, and T4) by using a pseudo-random number generating algorithm. Figure 1 presents the acquisition process for the study population.

The final study population of 210 patients (mean age, $64.4 \pm 9.3$ years; range, $35-84$ years) consisted of 146 men (mean age, $65.0 \pm 8.7$ years) and 64 women (mean age, $62.9 \pm 10.5$ years). The mean interval between CT and surgery was 15 days (range, $0-30$ days). The baseline characteristics are summarized in Table 1.

\section{Image Acquisition}

Chest CT was performed using one of the following scanners: Somatom Definition, Sensation-16 (Siemens Healthineers, Forchheim, Germany), LightSpeed 16, and Lightspeed VCT (GE Healthcare, Milwaukee, WI, USA), with settings of $120 \mathrm{kVp}$ and $150-200 \mathrm{mAs}$, pitch of $0.875-1$, and collimation of 1-1.25 mm. All CT data were acquired in the supine position with breathing suspended at full inspiration. Scan coverage was from the lung bases to the level of the thoracic inlet. Contrast enhancement was performed in all patients with $90-120 \mathrm{~mL}$ of intravenous iopromide (Ultravist, $300 \mathrm{mg} \mathrm{I} / \mathrm{mL}$; Bayer AG, Berlin, Germany) being injected at a rate of $3 \mathrm{~mL} / \mathrm{sec}$ using an automatic power injector, and scanning started after a delay of 50 seconds. CT images were reconstructed using a high-frequency algorithm. 


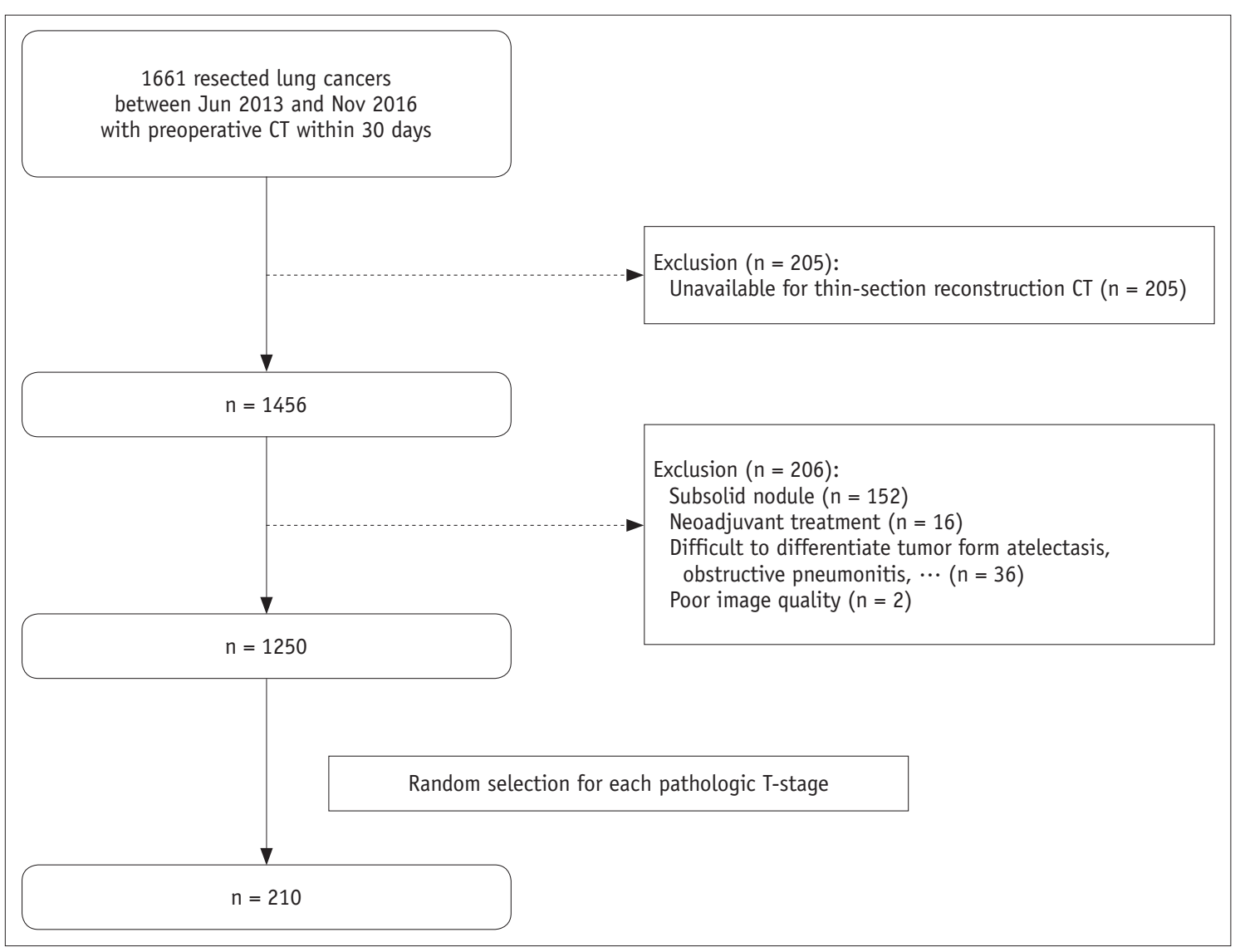

Fig. 1. Flow chart of patient inclusion procedure.

\section{Size Measurement of Lung Cancers on CT}

All CT data were anonymized and stored for review. Two readers (with 6 and 10 years of experience in thoracic radiology, respectively) who were blinded to pathologic size independently evaluated the 210 target lesions in the axial, coronal, and sagittal planes using a lung window setting (width, $1500 \mathrm{HU}$; level, -500 HU) (2) and commercial software (RadiAnt, Medixant, Poznan, Poland). The two readers were instructed to measure the axial diameter first and then measure the lesion on the other two planes in the order of their own preference at that time. Measurement of the MPR diameters required approximately 1 minute. For spiculated nodules, the spiculation was excluded from the measurement, while for cystic nodules the length of the largest nodular solid component was measured (2).

All diameters were measured using 0.1-mm increments, with the measurements then being rounded to the closest millimeter (2). The largest of the three diameter measurements was considered as the MPR diameter. Clinical T-stages were determined on the basis of axial and MPR diameters according to the size criteria in the eighth edition of the tumor node metastasis (TNM) classification.
Other T-descriptors were not considered in staging.

Measurement of the Longest Diameter on 3D by Using a Volumetrically Segmented Tumor Mask

To obtain the longest diameter of the 3D tumor volume, commercial software (Aview, Coreline Soft, Seoul, Korea) was used to segment the lesion on each section. The software automatically created an initial tumor mask, after which one radiologist manually corrected the volume of interest (VOI). Two radiologists confirmed the final VOI mask in consensus. The longest diameter within the VOI was then identified as the longest diameter on 3D images. This measurement required about 20-30 minutes per lesion.

\section{Pathologic Assessment}

All surgically resected lung specimens were formalinfixed and sectioned along their longest dimension. The longest diameter was then measured with a standard ruler and recorded in the pathologic report by two pathologists experienced in lung cancer assessments. The size in the pathologic report determined in routine clinical practice was used as the reference standard. Pathologic T-stages 
Table 1. Baseline Characteristics of Study Population $(n=210)$

\begin{tabular}{lc}
\hline \multicolumn{1}{c}{ Characteristics } \\
\hline Age, years, mean \pm SD & $64.4 \pm 9.3$ \\
Sex & \\
\hline Male & $146(69.5)$ \\
Female & $64(30.5)$ \\
Histologic subtype & \\
\hline Adenocarcinoma & $131(62.4)$ \\
\hline Lepidic & $5(2.4)$ \\
\hline Acinar & $29(13.8)$ \\
\hline Papillary & $62(29.5)$ \\
\hline Micropapillary & $1(0.5)$ \\
\hline Solid & $33(15.7)$ \\
\hline Mucinous & $1(0.5)$ \\
\hline Squamous cell carcinoma & $71(33.8)$ \\
\hline Small cell carcinoma & $7(3.3)$ \\
\hline Pleomorphic carcinoma & $1(0.5)$ \\
\hline Tumor size, mm, mean \pm SD & $39.2 \pm 25.4$ \\
Location & \\
\hline RUL & $57(27.1)$ \\
\hline RML & $10(4.8)$ \\
\hline RLL & $45(21.4)$ \\
\hline LUL & $44(21.0)$ \\
\hline LLL & $34(16.2)$ \\
\hline RUL, RML & $4(1.9)$ \\
\hline RML, RLL & $9(4.3)$ \\
\hline LUL, LLL & $7(3.3)$ \\
\hline
\end{tabular}

Data are presented as mean \pm SD or $n(\%)$, unless indicated otherwise. $\mathrm{LLL}=$ left lower lobe, $\mathrm{LUL}=$ left upper lobe, $\mathrm{RML}=$ right middle lobe, $\mathrm{RLL}=$ right lower lobe, $\mathrm{RUL}=$ right upper lobe, $\mathrm{SD}=$ standard deviation

were reassigned on the basis of only tumor size according to the eighth edition TNM classification.

\section{Statistical Analysis}

The mean axial and MPR diameters, longest diameter on 3D images, and pathologic sizes were calculated and compared using paired $t$ tests. Agreements between pathologic and CT measurements were assessed using BlandAltman plots and single-measure intraclass correlation coefficients (ICC). Inter-reader agreement was assessed by ICCs. The diagnostic accuracies of axial and MPR diameters for the determination of T-stage on CT were determined and compared using McNemar's test for the two readers according to the eighth edition of the lung cancer staging system $(1,12)$. Furthermore, to determine whether the effect of MPR measurement depends on the T-stages, T1a and T1b were grouped together into Group 1 and the other T-stages (T1c or higher) were grouped into Group 2. The diagnostic accuracies of axial and MPR diameters were compared within each group by using McNemar's test. The diagnostic accuracy of the longest diameter on 3D images was also evaluated.

Statistical analyses were performed using MedCalc statistical software (version 18.2.1; MedCalc Software bvba, Ostend, Belgium). Data are presented as mean \pm standard deviation. $P<0.05$ was considered to indicate statistical significance.

\section{RESULTS}

\section{Size Measurement on Pathology and CT}

The mean size of lung cancers on pathological assessments was $39.2 \pm 25.4 \mathrm{~mm}$. The mean sizes on axial and MPR images were $35.4 \pm 23.2 \mathrm{~mm}$ and $38.2 \pm 24.3 \mathrm{~mm}$, respectively, for reader 1 , and $36.8 \pm 23.5 \mathrm{~mm}$ and $40.0 \pm$ $25.1 \mathrm{~mm}$, respectively, for reader 2. MPR diameters were determined on 68 axial, 63 coronal, and 79 sagittal planes by reader 1, and on 94 axial, 54 coronal, and 62 sagittal planes by reader 2 . The longest diameter on $3 \mathrm{D}$ images was $44.6 \pm 27.7 \mathrm{~mm}$, which was significantly greater than the CT measurements reported by both readers and the pathologic diameter $(p<0.001$, all).

The axial diameters measured by each of the readers were significantly smaller than the pathologic diameters $(p<$ 0.001 , both). The MPR diameters measured by reader 1 were also significantly smaller than the pathologic diameters ( $p$ $=0.014)$, although there were no significant differences between the pathologic diameters and the MPR diameters of reader $2(p=0.180)$. The tumor measurements reported by reader 1 were significantly smaller than those reported by reader 2 on both axial and MPR CT ( $p<0.001$, both) (Table 2$)$.

The difference between the axial and pathologic diameters was $-3.9 \pm 6.6 \mathrm{~mm}$ for reader 1 and $-2.4 \pm 7.9 \mathrm{~mm}$ for reader 2 . The difference between the MPR and pathologic diameters was $-1.0 \pm 5.8 \mathrm{~mm}$ for reader 1 and $0.7 \pm 7 \mathrm{~mm}$ for reader 2. For both readers, the differences between MPR and pathologic diameters were significantly less than those between axial and pathologic diameters ( $p<0.001$, both).

The ICC between the MPR diameter of reader 1 and the pathologic diameter was the highest $(0.97 ; 95 \%$ confidence interval [CI], 0.96-0.98). The MPR and axial diameters of both readers showed a similar level of agreement with the pathologic diameter (0.97 [0.96-0.98] vs. 0.96 [0.95-0.97] for reader $1 ; 0.95$ [0.94-0.96] vs. 0.95 [0.93-0.96] for reader 2). The ICC between the pathologic diameter and 
Table 2. Size Measurement of Lung Cancer on CT by Two Readers and Pathologic Size Assessments

\begin{tabular}{|c|c|c|c|c|}
\hline \multirow{2}{*}{ Characteristics } & \multirow{2}{*}{ Mean Size $(\mathrm{mm})$} & \multicolumn{3}{|c|}{$P^{*}$} \\
\hline & & CT vs. Pathology & Axial vs. MPR & Reader 1 vs. 2 \\
\hline \multicolumn{5}{|l|}{ Reader 1} \\
\hline Axial diameter & $35.4 \pm 23.2$ & $<0.001$ & $<0.001$ & $<0.001$ \\
\hline MPR diameter & $38.2 \pm 24.3$ & 0.014 & & $<0.001$ \\
\hline \multicolumn{5}{|l|}{ Reader 2} \\
\hline Axial diameter & $36.8 \pm 23.5$ & $<0.001$ & $<0.001$ & \\
\hline MPR diameter & $40.0 \pm 25.1$ & 0.180 & & \\
\hline Pathology & $39.2 \pm 25.4$ & & & \\
\hline
\end{tabular}

Data are mean $\pm \mathrm{SD}$. *Paired $t$ test. MPR $=$ multiplanar reconstruction

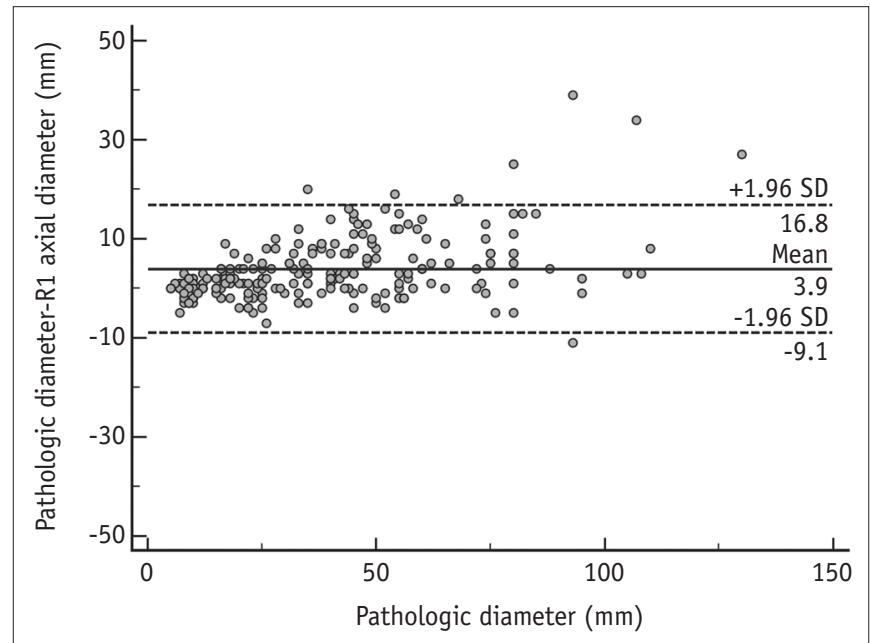

A

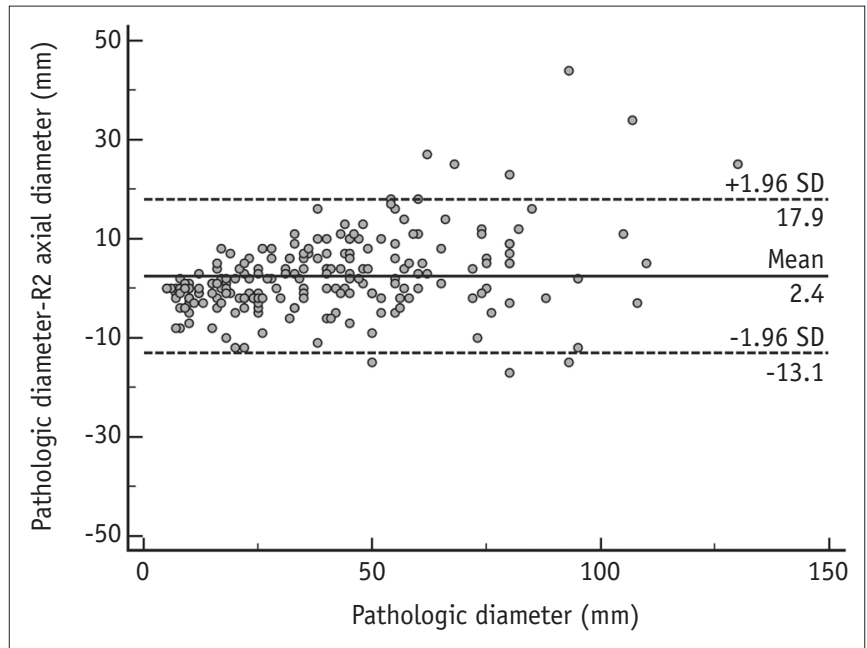

C

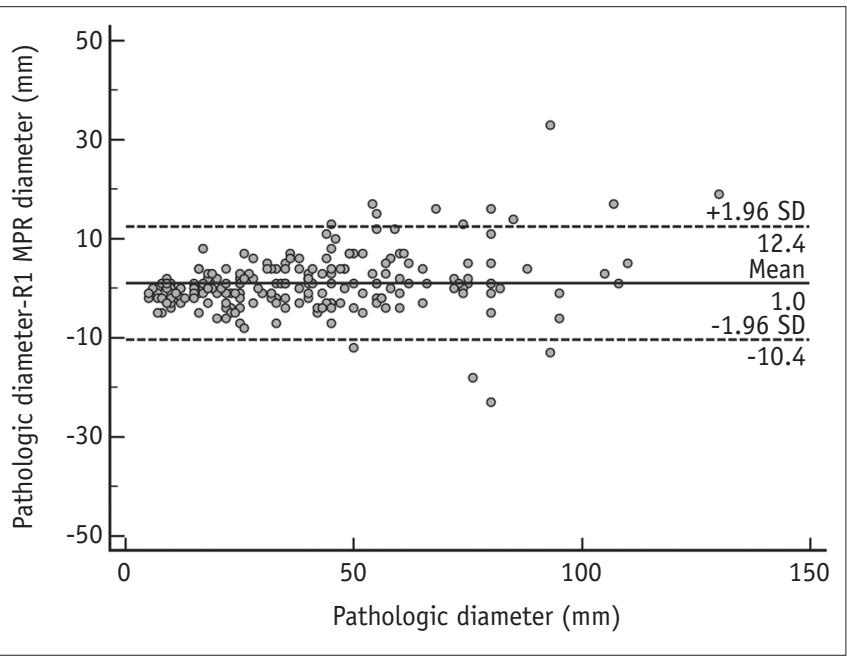

B

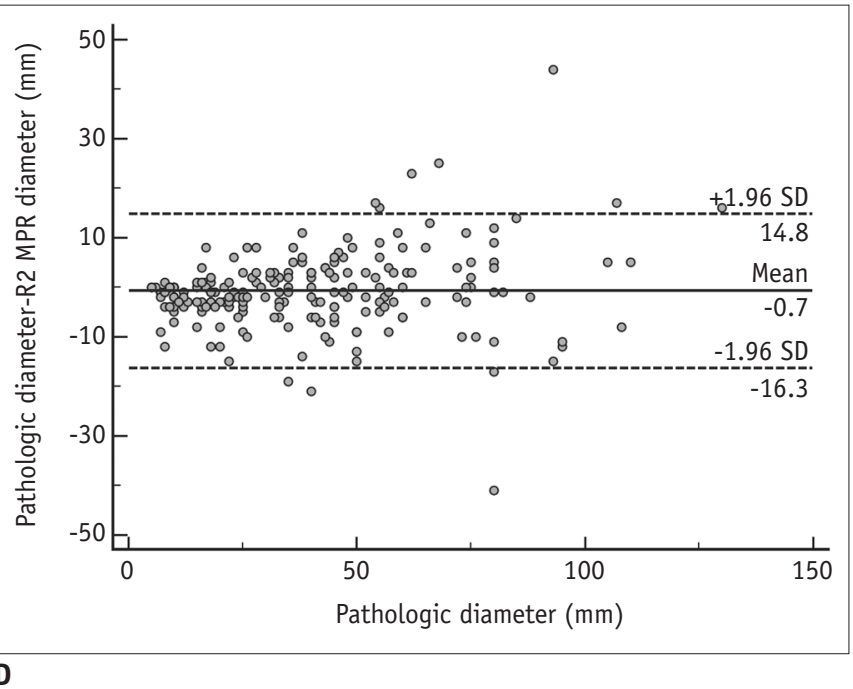

Fig. 2. Bland-Altman plots showing relationship between $\mathrm{CT}$ and pathologic diameters.

A-D. Graphs show differences between CT diameter and pathologic size. $\mathrm{X}$-axes represent pathologic size and $\mathrm{Y}$-axes represent differences in size between CT diameter and pathologic size. A. Axial diameter measured by reader 1. B. MPR diameter measured by reader 1 . C. Axial diameter measured by reader 2. D. MPR diameter measured by reader 2. MPR = multiplanar reconstruction, SD = standard deviation

the longest diameter in the 3D space was $0.95(0.93-0.96)$. The ICCs for interobserver agreement were $0.98(0.98-0.99)$ for the axial diameter and $0.98(0.97-0.99)$ for the MPR diameter.

Bland-Altman plots showing differences between pathologic and CT diameters are displayed in Figure 2. The 
Table 3. Comparison of T-Staging Accuracy on Different CT Planes

\begin{tabular}{lcccc}
\hline \multicolumn{1}{c}{ Staging Plane } & Accuracy & $P^{*}$ & Overstaging Rate & Understaging Rate \\
\hline Reader 1 (\%) & & 0.001 & & \\
$\quad$ Axial & $63.8(134 / 210)$ & & $11.9(25 / 210)$ & $30.0(63 / 210)$ \\
MPR & $74.3(156 / 210)$ & & $13.8(29 / 210)$ \\
Reader 2 (\%) & & 0.049 & $11.9(25 / 210)$ & $26.2(55 / 210)$ \\
Axial & $61.9(130 / 210)$ & & $18.6(39 / 210)$ & $13.3(28 / 210)$ \\
MPR & $68.1(143 / 210)$ & $41.4(87 / 210)$ & $3.8(8 / 210)$ \\
\hline
\end{tabular}

*McNemar's test. 3D = three-dimensional

95\% limits of agreement between the CT diameters reported by reader 1 and pathological assessments ranged from -9.1 $\mathrm{mm}$ to $16.8 \mathrm{~mm}$ (mean difference, $3.9 \mathrm{~mm}$ ) for axial diameter, and from $-10.4 \mathrm{~mm}$ to $12.4 \mathrm{~mm}$ (mean difference, $1.0 \mathrm{~mm}$ ) for MPR diameter. For reader 2, the $95 \%$ limits of agreement ranged from $-13.1 \mathrm{~mm}$ to $17.9 \mathrm{~mm}$ (mean difference, 2.4 $\mathrm{mm}$ ) for axial diameter, and from $-16.3 \mathrm{~mm}$ to $14.8 \mathrm{~mm}$ (mean difference, $-0.7 \mathrm{~mm}$ ) for MPR diameter. Differences between the longest diameter on $3 \mathrm{D}$ and the pathologic diameter showed the widest $95 \%$ limits of agreement (CI, $-22.2 \mathrm{~mm}$ to $11.4 \mathrm{~mm}$; mean difference, $-5.4 \mathrm{~mm}$ ).

\section{Diagnostic Accuracy of T-Staging}

The diagnostic accuracy of T-staging by axial diameter was $63.8 \%(134 / 210)$ for reader 1 and $61.9 \%$ (130/210) for reader 2. Overstaging using the axial plane alone was reported in $6.2 \%(13 / 210)$ and $11.9 \%(25 / 210)$ of cases for readers 1 and 2, respectively, whereas understaging was reported in $30.0 \%(63 / 210)$ and $26.2 \%(55 / 210)$ of cases. The diagnostic accuracy of T-staging by MPR diameter was $74.3 \%(156 / 210)$ and $68.1 \%(143 / 210)$ for readers 1 and 2 , respectively. When using MPR planes, $11.9 \%$ (25/210) and $18.6 \%(39 / 210)$ were overstaged by readers 1 and 2 , respectively, while $13.8 \%(29 / 210)$ and $13.3 \%(28 / 210)$ were understaged (Table 3 ).

For both readers, MPR diameter resulted in significant improvements in diagnostic accuracy compared with axial diameter alone ( $p=0.001$ for reader 1 , and $p=0.049$ for reader 2). When the T-stages were divided into two groups (Group 1, T1a/b; Group 2, T1c or higher), the diagnostic accuracy did not show any significant difference between MPR and axial diameters in Group 1 (73.3\% [44/60] vs. $81.7 \%$ [49/60] for reader $1 ; 63.3 \%$ [38/60] vs. $71.7 \%$ $[43 / 60]$ for reader $2 ; p=0.125$, both readers); however, MPR diameter had significantly better diagnostic accuracy than axial diameter in Group 2 (74.7\% [112/150] vs. 68.7\% [103/150] for reader $1, p<0.001 ; 70.0 \%$ [105/150] vs.
$58.0 \%$ [81/150] for reader 2, $p=0.001$ ) (Fig. 3). Table 4 presents the diagnostic accuracy of axial and MPR CT for each pathologic stage.

The longest diameter on 3D showed an accuracy of 54.8\% (115/210). Tumor measurements in 3D space resulted in overstaging in $41.4 \%$ of cases $(87 / 210)$, while $3.8 \%$ of patients $(8 / 210)$ were understaged. The diagnostic accuracy of the longest diameter on 3D was significantly lower than that of the MPR diameter for both readers $(p<0.001$ for reader $1, p=0.001$ for reader 2 ) (Table 3 ).

\section{DISCUSSION}

In this study, we demonstrated that size measurements on MPR CT significantly improved the diagnostic accuracy of T-staging, and the addition of 3D space data for lesion measurement did not improve the accuracy of T-staging.

There is an emphasis on lesion size for tumor staging in the eighth edition of the lung cancer staging classification. Accurate measurement of lesions on CT has therefore become more important for preoperative clinical staging. We found that the difference between the pathologic and $\mathrm{CT}$ diameters was significantly smaller when the diameter was measured on MPR images as opposed to axial images, suggesting that the MPR diameter is closer to the pathologic diameter and is more accurate than the axial diameter. These findings were linked to significant improvements in the diagnostic accuracy of T-staging. Therefore, we believe that MPR measurements are necessary for preoperative clinical staging to accurately assess lesion size and improve diagnostic accuracy.

More interestingly, the improvement in diagnostic accuracy depended on lesion size in our study. The diagnostic accuracy of the axial diameter was slightly higher than that of the MPR diameter in the T1a and T1b groups without statistical significance. In contrast, for the T1c or higher groups, MPR diameter resulted in significant 

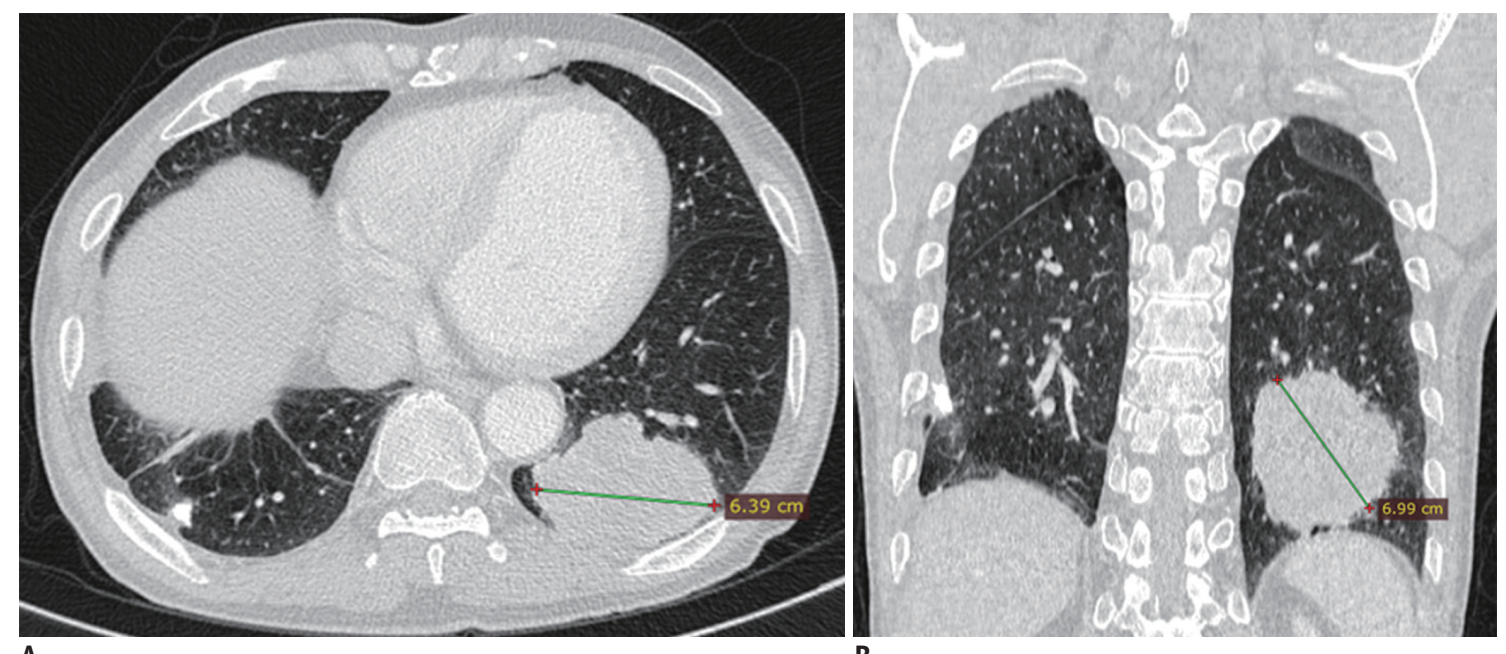

A
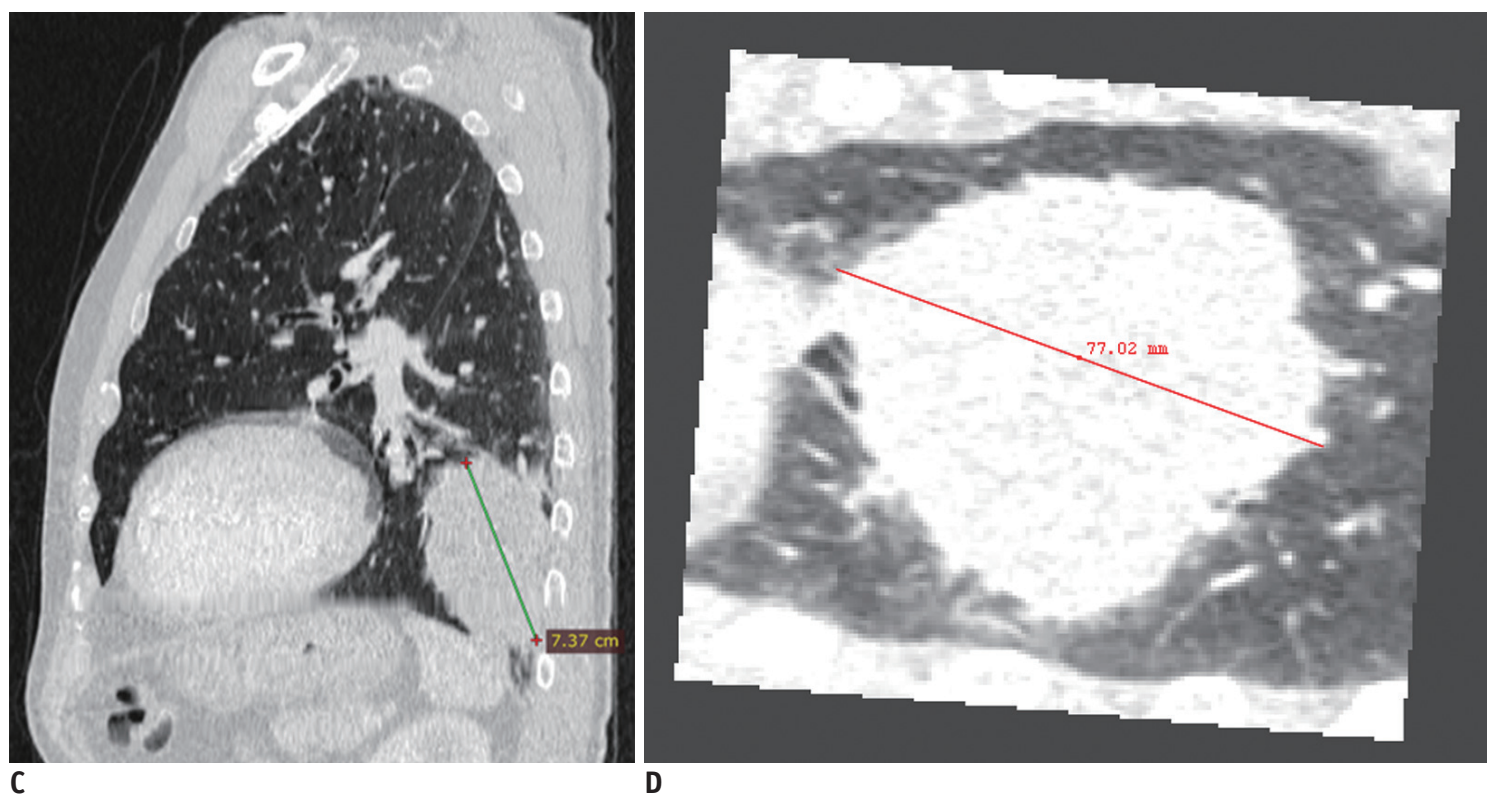

Fig. 3. Correct staging using MPR in T4 stages.

(A) Axial, (B) coronal, and (C) sagittal CT scans of large lobulated mass in left lower lobe of 74-year-old man. Tumor lies anterosuperiorly to posteroinferiorly, so longest dimension of tumor was captured on sagittal plane. On axial CT alone, both readers underestimated tumor size (64 mm and $62 \mathrm{~mm}$ ), with corresponding stage being T3. On sagittal CT, diameter measured by both readers was $74 \mathrm{~mm}$, which was equal to pathologic diameter and resulted in accurate clinical staging as T4. D. Longest diameter on three-dimensional was $77 \mathrm{~mm}$.

improvements in diagnostic accuracy for both readers. This is probably because larger tumors tend to grow unevenly in different directions, with accompanying parenchymal changes, and therefore evaluation with variable CT planes could help to accurately determine tumor extent.

Our results for the T1a and T1b subgroups correspond well with the results of the study by Ahn et al. (7). In their study, T-stages of 247 subsolid nodules were determined by the size of the solid portion according to the eighth edition TNM standards. Of the 247 lesions, 231 were T1b or lower, with only $16 \mathrm{~T} 1 \mathrm{c}$ lesions. They found that agreement between clinical and pathological T-staging was slightly better on axial measurements than on MPR measurements (50.0\% vs. $46.2 \%, p=0.084$ ).

Another issue concerning the application of MPR measurement is interobserver agreement. The chances of variability in the measurements would presumably be higher when three planes are used instead of one. Although Ridge et al. (6) did not estimate interobserver agreement for the largest MPR diameter, lower interobserver agreement (ICC) was found for the sagittal and coronal diameters than for the axial diameter ( 0.90 for axial, 0.87 for sagittal, and 0.75 
Table 4. Diagnostic Accuracy of Axial and MPR CT according to Pathologic Stage

\begin{tabular}{|c|c|c|c|c|c|c|}
\hline \multirow{2}{*}{$\begin{array}{c}\text { Pathologic } \\
\text { T Stage }\end{array}$} & \multicolumn{3}{|c|}{ Reader $1(\%)$} & \multicolumn{3}{|c|}{ Reader $2(\%)$} \\
\hline & Axial & MPR & Axial vs. MPR* & Axial & MPR & Axial vs. MPR* \\
\hline T1a & $73.3(22 / 30)$ & $63.3(19 / 30)$ & \multirow{2}{*}{$p=0.125$} & $63.3(19 / 30)$ & $60.0(18 / 30)$ & \multirow{2}{*}{$p=0.125$} \\
\hline T1b & $90.0(27 / 30)$ & $83.3(25 / 30)$ & & $80.0(24 / 30)$ & $66.7(20 / 30)$ & \\
\hline T1c & $66.7(20 / 30)$ & $80.0(24 / 30)$ & \multirow{5}{*}{$p<0.001$} & $70.0(21 / 30)$ & $76.7(23 / 30)$ & \multirow{5}{*}{$p=0.001$} \\
\hline T2a & $50.0(15 / 30)$ & $66.7(20 / 30)$ & & $46.7(14 / 30)$ & $63.3(19 / 30)$ & \\
\hline $\mathrm{T} 2 \mathrm{~b}$ & $36.7(11 / 30)$ & $63.3(19 / 30)$ & & $43.3(13 / 30)$ & $53.3(16 / 30)$ & \\
\hline T3 & $70.0(21 / 30)$ & $83.3(25 / 30)$ & & $60.0(18 / 30)$ & $73.3(22 / 30)$ & \\
\hline T4 & $60.0(18 / 30)$ & $80.0(24 / 30)$ & & $70.0(21 / 30)$ & $83.3(25 / 30)$ & \\
\hline
\end{tabular}

Data are mean \pm SD. * McNemar's test.

for coronal plane). However, our results showed almost the same interobserver agreement (ICC, 0.98 vs. 0.98 ) for MPR and axial-only measurements. Ahn et al. (7) showed similar results for interobserver agreement ( 0.83 vs. 0.82 for MPR and axial diameter, respectively).

In this study, we also evaluated whether the longest diameter on $3 \mathrm{D}$ could yield results closer to the pathologic size. Although off-axis oblique reformations are not currently recommended because of poor reproducibility (2), they could become more reproducible and applicable with advances in techniques. However, according to our results, adding the longest diameter on $3 \mathrm{D}$ to the T-staging did not improve the diagnostic accuracy and instead reduced it.

Theoretically, the longest diameter on 3D would be the closest to the pathologic diameter, but the reality is different because of the inherent limitations of CT. Since CT does not always allow the differentiation of tumor from associated atelectasis, inflammation, surrounding edema, and even adjacent mucus impaction, CT measurements tend to overestimate tumor size in comparison with pathologic size, as shown in several previous studies (1315). Furthermore, the in vivo state of a tumor in inflated lung tissues on CT could be slightly different to that of the pathological resected specimen, which may have shrunk and deformed during tissue processing. It is also possible that tumor parts could be lost or impaired during the operation. Consequently, our results support the opinion in the Fleischner Society guidelines that the additional effort to find the longest diameter in the off-axis plane is not currently recommended.

Our study has several limitations. First, our study had a retrospective design, and therefore it may be subject to bias. Second, we only included solid lesions. However, as T-staging is determined by the size of the solid portion even if the lesion includes ground glass opacity, our study could also be applicable to part-solid lesions. Furthermore,
Ahn et al. (7) presented similar results to ours. Third, our study used the same number of patients for each pathologic T-stage, which is different from the natural incidence. Although the natural incidences of T-stages can vary according to time and place, further external validation of our results is needed. Fourth, our study addressed the accuracy of T-stages only on the basis of tumor size and did not consider other T-descriptors such as invasion to adjacent structures or separate nodules. However, in our study population, only 19 of 210 patients showed upstaging due to other T-descriptors, mostly visceral pleural invasion. Finally, since our study used the pathologic diameter as a reference standard, it is unclear whether the MPR diameter better reflects patient prognosis or affects treatment decision. Therefore, further studies are warranted to determine the association between MPR measurements and clinical outcomes.

In conclusion, size measurement on MPR CT can help to accurately determine preoperative clinical T-stages in lung cancers without impairing interobserver agreement. The size in the 3D space showed no added value.

\section{Conflicts of Interest}

The authors have no potential conflicts of interest to disclose.

\section{ORCID iDs}

Sang Min Lee https://orcid.org/0000-0001-7627-2000

Sohee Park

https://orcid.org/0000-0002-4009-1571

Jooae Choe

https://orcid.org/0000-0003-0486-4626

June-Goo Lee

https://orcid.org/0000-0002-1380-6682 
Sang Min Lee

https://orcid.org/0000-0002-2173-2193

Kyung-Hyun Do

https://orcid.org/0000-0003-1922-4680

Joon Beom Seo

https://orcid.org/0000-0003-0271-7884

\section{REFERENCES}

1. Goldstraw P, Chansky K, Crowley J, Rami-Porta R, Asamura H, Eberhardt WE, et al.; International Association for the Study of Lung Cancer Staging and Prognostic Factors Committee, Advisory Boards, and Participating Institutions. The IASLC lung cancer staging project: proposals for revision of the TNM stage groupings in the forthcoming (eighth) edition of the TNM classification for lung cancer. J Thorac Oncol 2016;11:39-51

2. Bankier AA, MacMahon H, Goo JM, Rubin GD, Schaefer-Prokop CM, Naidich DP. Recommendations for measuring pulmonary nodules at CT: a statement from the Fleischner Society. Radiology 2017;285:584-600

3. Oxnard GR, Zhao B, Sima CS, Ginsberg MS, James LP, Lefkowitz RA, et al. Variability of lung tumor measurements on repeat computed tomography scans taken within 15 minutes. J Clin Oncol 2011;29:3114-3119

4. Heidinger BH, Anderson KR, Moriarty EM, Costa DB, Gangadharan SP, VanderLaan PA, et al. Size measurement and T-staging of lung adenocarcinomas manifesting as solid nodules $\leq 30 \mathrm{~mm}$ on CT: radiology-pathology correlation. Acad Radiol 2017;24:851-859

5. Heidinger BH, Nemec U, Anderson KR, Costa DB, Gangadharan SP, VanderLaan PA, et al. "Rounding" the size of pulmonary nodules: impact of rounding methods on nodule management, as defined by the 2017 Fleischner Society guidelines. Acad Radiol 2017;24:1422-1427

6. Ridge CA, Huang J, Cardoza S, Zabor EC, Moskowitz CS, Zakowski MF, et al. Comparison of multiplanar reformatted CT lung tumor measurements to axial tumor measurement alone: impact on maximal tumor dimension and T stage. AJR Am J
Roentgenol 2013;201:959-963

7. Ahn H, Lee KW, Lee KH, Kim J, Kim K, Chung JH, et al. Effect of computed tomography window settings and reconstruction plane on 8th edition T-stage classification in patients with lung adenocarcinoma manifesting as a subsolid nodule. Eur J Radiol 2018;98:130-135

8. Wang Y, de Bock GH, van Klaveren RJ, van Ooyen P, Tukker W, Zhao $Y$, et al. Volumetric measurement of pulmonary nodules at low-dose chest CT: effect of reconstruction setting on measurement variability. Eur Radiol 2010;20:1180-1187

9. Goo JM, Tongdee T, Tongdee R, Yeo K, Hildebolt CF, Bae KT. Volumetric measurement of synthetic lung nodules with multi-detector row CT: effect of various image reconstruction parameters and segmentation thresholds on measurement accuracy. Radiology 2005;235:850-856

10. Petrou M, Quint LE, Nan B, Baker LH. Pulmonary nodule volumetric measurement variability as a function of CT slice thickness and nodule morphology. AJR Am J Roentgenol 2007;188:306-312

11. Ravenel JG, Leue WM, Nietert PJ, Miller JV, Taylor KK, Silvestri GA. Pulmonary nodule volume: effects of reconstruction parameters on automated measurements--a phantom study. Radiology 2008;247:400-408

12. Detterbeck FC, Boffa DJ, Tanoue LT. The new lung cancer staging system. Chest 2009;136:260-271

13. Lee KH, Goo JM, Park SJ, Wi JY, Chung DH, Go H, et al. Correlation between the size of the solid component on thinsection CT and the invasive component on pathology in small lung adenocarcinomas manifesting as ground-glass nodules. $J$ Thorac Oncol 2014;9:74-82

14. Lampen-Sachar K, Zhao B, Zheng J, Moskowitz CS, Schwartz LH, Zakowski MF, et al. Correlation between tumor measurement on computed tomography and resected specimen size in lung adenocarcinomas. Lung Cancer 2012;75:332-335

15. Stroom J, Blaauwgeers $H$, van Baardwijk A, Boersma L, Lebesque J, Theuws J, et al. Feasibility of pathologycorrelated lung imaging for accurate target definition of lung tumors. Int J Radiat Oncol Biol Phys 2007;69:267-275 